\title{
Some thoughts on bioimpedance and biodielectrics
}

\author{
Sverre Grimnes and Ørjan G. Martinsen \\ 1. Editors-in-chief, Journal of Electrical Bioimpedance \\ 2. Department of Physics, University of Oslo, Oslo, Norway \\ 3. Department of Biomedical and Clinical Engineering, Rikshospitalet, Oslo University Hospital, Oslo Norway
}

From early on we learnt that a dielectric is an insulator, and a practical way to measure it was to place it between two capacitor plates. The plates were dry and so was the dielectric - the resistance between the plates was very high. With living tissue it was quite different, the material was not dry and the capacitor plates had wet contact with the tissue. At the wet plates polarization impedance appeared constituting an important source of error. The discipline of bioimpedance had one problem more than the discipline of dielectrics. Even so it became more and more common to classify tissue as a dielectric instead of a conductor. The terms melted together, but not completely. Taking a closer look at the theory of Bioimpedance and Biodielectrics we discover that both disciplines suffer from a lack of consistency.

Dielectric theory emerged much earlier than impedance theory; it started more than 200 years ago with Coulomb's law (1795):

$$
F=k_{e} \frac{q_{1} q_{2}}{r^{2}}
$$

where $\mathrm{k}_{\mathrm{e}}$ is the Coulomb's constant. It had a large impact on the global choice of System of units. In the old cgs system $\mathrm{k}_{\mathrm{e}}=1$ (dimensionless), in the present SI system $\mathrm{k}_{\mathrm{e}}=1 / 4 \pi \varepsilon_{0}$. The law implied that the science of electro-statics early had a sound theoretical basis, and this was taken into account by Maxwell who around 1875 introduced the three basic invariant quantities of free space related according to $\mathrm{c}_{0}^{2} \mu_{0} \varepsilon_{0}=1$. Here $\mathrm{c}_{0}$ is the speed of light, $\mu_{0}$ the magnetic permeability and $\varepsilon_{0}$ the permittivity, all three related to free space. The equation shows that the speed of light is embedded in Coulomb's law. This presupposes that light is electromagnetic waves, so that e.g. optical spectroscopy may take over from dielectric spectroscopy at the highest frequencies.

In the 3.ed edition of Maxwells $A$ treatise on electricity and magnetism (1891) the terms permittivity and admittance were still not used. Maxwell measured on e.g. glasses and electrolytes but was not focusing on living materials, even if he analyzed electrolytic suspensions with glass spheres and even coated glass spheres. Maxwell classified dry materials as dielectrics, but electrolytes as conductors.

The term admittance based upon sinusoidal signals was coined by Heaviside, 100 years after Coulomb's law. Generation of phase shifts in the sine waveforms made it necessary to consider many variables in complex form, e.g. complex permittivity $\boldsymbol{\varepsilon}=\varepsilon^{\prime}-\varepsilon^{\prime \prime}$, complex admittance $\mathbf{Y}=\mathrm{G}+\mathrm{jB}$ and complex impedance $\mathbf{Z}=\mathrm{R}+\mathrm{j} X$.
Høber (1911) made early impedance measurements at different frequencies and showed the existence of cell membranes. The Debye (1913) model was based upon exponential relaxation with ideal resistors and capacitors. Cole (1928) pioneered both permittivity and impedance measurements on living cell suspensions, tissue and nerves in particular. However, in the 1920ies it became more and more clear that most dielectrics are not in accordance with the Debye model because they do not show exponential time relaxation. The use of exponential relaxation data was to a large extent replaced by sinusoidal frequency spectrum analysis as a result of technology advancement. K.S. Cole started to publish results using complex plane presentation, and the Cole brothers in 1941 published a break-through article on dielectrics and permittivity with the dispersion equation:

$$
\varepsilon=\frac{\varepsilon_{s}-\varepsilon_{\infty}}{1+\left(j \omega \tau_{0}\right)^{1-\alpha}}
$$

However, the equations were empirical and in addition with a problematic use of relaxation theory and the enigmatic parameters $\alpha$ and $\tau$. Living systems were hardly mentioned and relaxation theory with non-exponential behavior were put aside. Actually K.S. Cole alone had already in 1940 published the bioimpedance version of dispersion before the Cole-Cole permittivity equations:

$$
Z=R_{\infty}+\frac{R_{0}-R_{\infty}}{1+\left(j \omega \tau_{z}\right)^{\alpha}}
$$

It was clear to everyone that in the Cole and Cole-Cole equations the exponent symbol $0<|\alpha|<1$ used fractional power laws not in accordance with exponential relaxation behavior. The equations were empirical, and contained a time constant $\tau$. It was proposed that this represents a spread of relaxation times (which was natural regarding the complexity of living tissue). Another proposal was the existence of a constant phase element (CPE) somewhere in the system. Other non-accordance findings were inductivecapacitive phenomena (Cole and Baker,1941). That was a strange finding, in particular because they found that the inductive properties were situated in the extremely thin cell membrane. An additional problem was that the Cole and Cole-Cole equations were based on AC phenomena and it was difficult to introduce DC parameters in the models.

JEB has registered both the strong common basis and the lack of theory-based models in our two disciplines. 